\title{
Alkaloid Composition of the Nicotiana Species *
}

\author{
by
}

Verne A. Sisson

Crops Research Laboratory, Agricultural Research Service, United States Department of Agriculture, Oxford, N.C., U.S.A., and

North Carolina Agricultural Research Service, North Carolina State University,

Raleigh, N.C., U.S.A.

and

\section{R. F. Severson}

Tobacco Quality and Safety Research Unit, Richard B. Russell Agricultural Research Center, Agricultural Research Service, United States Department of Agriculture,

Athens, Georgia, U.S.A.

\section{SUMMARY}

Plants from 64 Nicotiana species were sampled in the greenhouse in 1983 and 1984 and from the field in 1985 and 1986 for the purpose of developing a chemical profile of each species. Mature green leaves were harvested at flowering, freeze-dried and ground to pass a $1 \mathrm{~mm}$ screen prior to chemical analysis. In this study we report the type and amounts of nicotinoid alkaloids. Alkaloid values were determined by glass-capillary gas chromatography and were averaged over the two years for each environment. Both total alkaloids ( $\mathrm{mg} \mathrm{g}^{-1}$ dry weight) and the distribution (percentage composition) of nicotine, nornicotine, anabasine, and anatabine were highly correlated between years for greenhouse and field samples. Greenhouse and field data were highly correlated, although total-alkaloid levels were significantly higher from field-grown plants. All of the Nicotiana species tested contained a measurable alkaloid fraction (at least $10 \mu \mathrm{g} \mathrm{g}^{-1}$ ). There was a wide range in total-alkaloid levels with nearly a 200-fold difference

\footnotetext{
* Received: 10th May 1989 - accepted: 21st July 1989.

Paper No. 12130 of the Journal Series of the North Carolina Agricultural Research Service, Raleigh, N.C.

The use of trade names in this publication does not imply endorsement by the North Carolina Agricultural Research Service or the U.S. Department of Agriculture of the products named, nor criticism of similar ones not mentioned.
}

among greenhouse-grown samples and a 400 -fold difference among field-grown species. In general, total-alkaloid levels among the species were found to be relatively low. Eleven species were found to have a different alkaloid composition when green leaf and air-dried samples were compared. Evidence is presented for the association between alkaloid characteristics and the phylogenetic classification of the species in the genus.

\section{ZUSAMMENFASSUNG}

Anhand von Pflanzenmaterial, das in den Jahren 1983 und 1984 im Gewächshaus und in den Jahren 1985 und 1986 im Feldanbau gezogen worden war, wurden 64 Nicotiana-Arten einzeln auf ihre chemische Zusammensetzung hin untersucht. Zum Zwecke der qualitativen und quantitativen Bestimmung der Nicotinalkaloide wurde das reife grüne Blattgut im Stadium der Blüte geerntet, gefriergetrocknet, gemahlen und gesiebt $(<1 \mathrm{~mm})$. Die Analyse erfolgte gaschromatographisch unter Einsatz von Glaskapillarsäulen, und die Ergebnisse werden für beide Anbauarten als Durchschnittswerte der zwei jeweiligen Jahre dargelegt. Die Werte für Gesamtalkaloide ( $\mathrm{mg} \mathrm{g}^{-1}$ Trockenmasse) wie auch jene für die Verteilung, d. h. den prozentualen Anteil 
der einzelnen Verbindungen (Nicotin, Nornicotin, Anabasin und Anatabin) waren im Vergleich zwischen den einzelnen Anbauperioden bzw. Anbauarten in hohem Maße miteinander korreliert. Es bestand eine gute Korrelation zwischen den Werten der Gewächshausund jenen der Feldanbauproben, obwohl der Gesamtalkaloidgehalt bei den im Feldanbau gezogenen Pflanzen signifikant höher war. Alle untersuchten NicotianaArten wiesen eine meßbare Alkaloidfraktion auf (wenigstens $10 \mu \mathrm{g} \mathrm{g}^{-1}$ ). Der Gehalt an Gesamtalkaloiden war sehr unterschiedlich; die Werte differierten vonein-

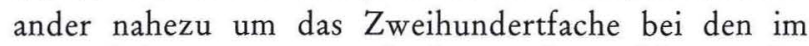
Gewächshaus gezogenen Proben und ungefähr um das Vierhundertfache bei den im Feld angebauten Proben, wobei die Gesamtalkaloidmengen durchweg verhältnismäßig gering waren. Bei elf Arten war die Alkaloidzusammensetzung unterschiedlich, wenn grünes Blattgut und luftgetrocknete Proben miteinander verglichen wurden. Zwischen der charakteristischen Alkaloidzusammensetzung und der phylogenetischen Klassifizierung der Art innerhalb der Gattung ließ sich ein $\mathrm{Zu}-$ sammenhang nachweisen.

\section{RESUME}

La composition chimique de chacune de 64 espèces de Nicotiana a été étudiée à l'aide d'échantillons prélevés sur des plantes ayant été cultivées en serre en 1983 et 1984 et en plein champ en 1985 et 1986 . En vue de l'analyse qualitative et quantitative des alcaloïdes nicotiniques, les feuilles vertes à maturation ont été récoltées au moment de la floraison, lyophilisées, moulues et tamisées $(<1 \mathrm{~mm})$. L'analyse a été effectuée par chromatographie en phase gazeuse en utilisant des colonnes capillaires en verre. Les résultats indiqués sont les valeurs moyennes correspondant aux deux années pour chaque type de culture. Les teneurs en alcaloïdes totaux ( $\mathrm{mg} / \mathrm{g}$ de matière sèche) de même que les pourcentages respectifs des différents composés (nicotine, nornicotine, anabasine et anatabine) apparaissent fortement corrélés avec les périodes et les types de culture. Les valeurs obtenues pour les échantillons provenant des serres sont bien corrélées avec celles correspondant aux échantillons des plantes cultivées en plein champ bien que la teneur en alcaloïdes totaux de ces dernières soit nettement plus élevée. Toutes les espèces de Nicotiana examinées contenaient une fraction d'alcaloïdes mesurable (au moins $10 \mu \mathrm{g} / \mathrm{g}$ ). Les teneurs en alcaloïdes totaux se sont avérées très variables: les résultats trouvés varient dans un rapport de 1 à 200 pour les échantillons provenant des serres et de 1 à 400 pour ceux provenant des plantes cultivées en plein champ, les quantités d'alcaloïdes totaux étant en général relativement faibles. Dans le cas de onze espèces, on a observé que la composition en alcaloïdes différait selon qu'il s'agissait d'une feuille verte ou d'un échantillon séché à l'air. Il a été possible de mettre en évidence un rapport entre la teneur caractéristique en alcaloïdes et la classification phylogénétique de l'espèce au sein du genre.

\section{INTRODUCTION}

The nicotine alkaloids are characteristic compounds in the chemistry of the Nicotiana genus. Although alkaloids are apparently active metabolites in tobacco (17), their usefulness to most plants remains uncertain (8). However, the presence of alkaloids in all Nicotiana species is an indication that they may have had some adaptive value to the genus as a whole (14).

The alkaloid composition of the Nicotiana species has been the subject of several investigations $(4,9,10,11$, $15,16)$. The principal interest in these works was the kind and amount of alkaloids found in each species. The findings of these independent investigations were in good agreement: [1] all observed wild species contained one or more alkaloids, [2] generally, a single alkaloid was found to predominate in a given species, [3] nicotine or nornicotine occurred as the main alkaloid in most species, but a few species produce anabasine as the principal alkaloid, [4] total-alkaloid levels were found to be highly variable, and [5] no clearly defined associations between phylogenetic position and the kinds or amounts of alkaloids was evident.

Various techniques for isolation and analysis of alkaloids were used in these investigations. The methods provided accurate measures of total alkaloids, but with the exception of the work by SAITOH et al. (9) and SARYCHEV (10) quantitative estimates of individual alkaloids were much less precise. Quantitative determinations can be important in determining the genetic and evolutionary association among species, as well as understanding the biochemical processes involved in alkaloid production.

This investigation represents the most comprehensive characterization of the alkaloid content of the Nicotiana species to date. All of the currently available $\mathrm{Ni}$ cotiana species were evaluated using modern analytical techniques to provide quantitative and qualitative measures of the individual alkaloids present in each species. Furthermore, the analyses are unique in that they cover multiple years and environments and that all species were evaluated for alkaloid composition in each year the investigations were conducted.

This paper deals mainly with the alkaloid chemistry of the Nicotiana species and, therefore, the taxonomy of the genus will not be discussed in detail. Readers are referred to Goodspeed (2) and SMITH (14) for a taxonomic classification of the genus, as well as author citations for each species.

\section{MATERIALS AND METHODS}

\section{Cultural Methods}

Seeds of the Nicotiana species came from the National Plant Germplasm System's Nicotiana Species Germplasm Collection maintained at the Crops Research Laboratory of the U.S. Department of Agriculture Agricul- 
tural Research Service, Oxford, North Carolina. A total of 66 species, including several of tentative species status, were grown for sampling each year from 1983 through 1986. The first two years plants were grown in a greenhouse environment. Forty to fifty seeds of each species were sown on a sterile medium of coarse vermiculite in $10 \mathrm{~cm}$ clay pots in early March. When individual seedlings of each species attained a size of $2 \mathrm{~cm}$ to $4 \mathrm{~cm}$, they were transplanted into separate $15 \mathrm{~cm}$ pots containing a soil/peat/sand $(1 / 1 / 1(\mathrm{v} / \mathrm{v} / \mathrm{v}))$ mixture. Plants were grown in the greenhouse without supplemental lighting. Temperatures during the growing period were maintained at about $27{ }^{\circ} \mathrm{C}$ during the daytime and $18{ }^{\circ} \mathrm{C}$ at night. A standard fertilizer mix was applied weekly to promote vigorous, healthy plant development. When a species flowered, the inflorescence and all suckers and side branches were removed. Days to flower varied among the species from 68 to 207 days after seeding. Seven days after plants were topped and suckered all mature green leaves were harvested, the midveins were excised, and the laminar sample was immediately frozen. Several short-day species had not flowered after 207 days but were sampled at that time. The number of plants sampled per species varied from as many as 30 for the smaller-statured species to a minimum of five plants for the largest species. The green weight for samples varied between $150 \mathrm{~g}$ to $300 \mathrm{~g}$. The frozen-laminae samples were later lyophilized and ground in a Wiley mill to pass a $1 \mathrm{~mm}$ mesh screen. Samples were stored in polyethylene bags at $10^{\circ} \mathrm{C}$ prior to chemical analysis.

In 1985 and 1986 plants were grown in the field. Seeds were germinated in the greenhouse as previously described. Small seedlings were transplanted into $5 \times$ $5 \mathrm{~cm}$ cells in styrofoam flats containing a 50:50 mixture of Redi-Earth ${ }^{\circledR}$ and fine-textured vermiculite. In May, the seedlings were handtransplanted to two replicated field plots. The field was fumigated with methyl bromide prior to transplanting and a broadcast application of $45.5 \mathrm{~kg}$ of $16-0-0 \mathrm{NPK}$ fertilizer was made. Mature green leaves were harvested from each plot at the time of flowering. Plants were not topped and suckered as had been done in the greenhouse. The green-leaf samples from the field were processed for chemical analysis in the same manner as the greenhouse samples.

In 1984 and 1986 duplicate green-leaf samples were taken from 25 species which were identified as producing predominantly nicotine. Leaves making up this duplicate sample were threaded on strings, hung on sticks, and allowed to dry naturally under ambient conditions (air cure). Normal drying time was from three to four weeks for the different species. Midveins were stripped from the dried leaves and the laminae ground in a manner identical to the freeze-dried sample.

\section{Analytical Procedures}

A rapid glass-capillary gas-chromatographic (GC) method (13) was used for the analysis of nicotine, nornicotine, anabasine, anatabine, and myosmine (and in 1984,85 and 86 samples, $N$-acetylnornicotine) present in the Nicotiana species. About $25 \mathrm{mg}$ to $50 \mathrm{mg}$ of the ground laminar sample and $1 \mathrm{ml}$ of $0.05 \mathrm{M}$ methanolic potassium hydroxide containing the internal standard, $2,4^{\prime}$-dipyridyl, were combined in a standard $2 \mathrm{ml}$ autosampler vial, and the vial was capped and placed in an ultrasonic bath for $15 \mathrm{~min}$. The samples were analyzed with a GC equipped with an automatic sampler and a nitrogen-phosphorus detector (NPD). The alkaloids were analyzed by using 60:1 split injection on a $0.32 \mathrm{~mm}$ inside diameter $\times 15 \mathrm{~m}$ Superox ${ }^{(\mathrm{m})}-4$ fusedsilica column (temperature program: $120^{\circ} \mathrm{C}$ to $190{ }^{\circ} \mathrm{C}$ at $4^{\circ} / \mathrm{min}, 190{ }^{\circ} \mathrm{C}$ to $240{ }^{\circ} \mathrm{C}$ at $8^{\circ} / \mathrm{min}$; linear flow velocity: $35 \mathrm{~cm} / \mathrm{s}$ of $\mathrm{He}$ ). The injection-port temperature was $200{ }^{\circ} \mathrm{C}$, the detector temperature was $280^{\circ} \mathrm{C}$, and $2 \mu \mathrm{l}$ samples were injected. The NPD-response curves were obtained by analysis of a series of standard solutions containing nicotine, nornicotine, anabasine, 2,4'dipyridyl and $\mathrm{N}$-acetylnornicotine. Anatabine was assumed to respond like anabasine. The $\mathrm{N}$-acetylnornicotine was prepared and isolated as described by SEVERson et al. (12). The alkaloid components in N. tabacum, $N$. repanda and $N$. glauca were characterized by GC/ MS. The alkaloid components for all other samples were identified by GC retention data only.

\section{RESULTS}

Using a modern gas-chromatographic method for the analysis of the nicotine alkaloids, a quantitative determination of the major alkaloid components of all but two of the currently recognized Nicotiana species was made. The species $N$. ameghino $i$ and $N$. longibracteata are known only from herbarium specimens and seeds are not available. Total-alkaloid levels $\left(\mathrm{mg} \mathrm{g}^{-1}\right.$ dry weight) and individual alkaloid-component distribution (percentage composition) from 64 species and 2 entries of tentative species rank are presented in Table 1 (1983-1984 greenhouse), and Table 2 (1985-1986 field). These results include the analysis of $N$. acaulis and $N$. linearis, two species for which alkaloid content have never been reported.

Both total alkaloids ( $\mathrm{mg} \mathrm{g}^{-1}$ dry weight) and the distribution (percentage composition) of nicotine, nornicotine, anabasine, and anatabine were highly correlated between years for greenhouse and field samples. Furthermore, greenhouse and field data were highly correlated, although total-alkaloid levels were significantly higher in field-grown plants.

All of the Nicotiana species tested contained a measurable alkaloid fraction (at least $10 \mu \mathrm{g} \mathrm{g}^{-1}$ ). There was a wide range in total-alkaloid levels with nearly a 200fold difference among greenhouse-grown samples and a 400-fold difference among field-grown species. In general, total-alkaloid levels among the species were found to be relatively low. 
Table 1.

Alkaloid levels and composition of the Nicotiana species, from greenhouse-grown plants.

\begin{tabular}{|c|c|c|c|c|c|c|c|}
\hline \multirow{2}{*}{$\begin{array}{l}\text { Subgenus } \\
\text { Section } \\
\text { Species }\end{array}$} & \multirow{2}{*}{$\begin{array}{c}\text { Total } \\
\text { alkaloids* } \\
(\mathrm{mg} / \mathrm{g})\end{array}$} & \multicolumn{5}{|c|}{ Percentage composition } & \multirow{2}{*}{$\begin{array}{c}N \text {-acetyl- } \\
\text { nornicotine } \\
(\mathrm{mg} / \mathrm{g})\end{array}$} \\
\hline & & $\begin{array}{c}\text { Nico- } \\
\text { tine }\end{array}$ & $\begin{array}{c}\text { Nornico- } \\
\text { tine }\end{array}$ & $\begin{array}{l}\text { Anab- } \\
\text { asine }\end{array}$ & $\begin{array}{l}\text { Anat- } \\
\text { abine }\end{array}$ & $\begin{array}{c}\text { Myos- } \\
\text { mine }\end{array}$ & \\
\hline \multicolumn{8}{|l|}{ Rustica } \\
\hline \multicolumn{8}{|l|}{ Paniculatae } \\
\hline glauca & 4.2 & 1.8 & 0.3 & 97.6 & 0.3 & n.d. ${ }^{+}$ & n.d. \\
\hline paniculata & 3.8 & 90.6 & 9.2 & 0.1 & 0.1 & trace & trace $^{++}$ \\
\hline knightiana & 4.0 & 92.6 & 4.1 & 3.0 & 0.3 & n.d. & n.d. \\
\hline solanifolia & 3.8 & 0.7 & 53.8 & 45.3 & 0.2 & n.d. & 0.03 \\
\hline benavidesii & 6.4 & 90.0 & 1.3 & 8.0 & 0.7 & n.d. & n.d. \\
\hline cordifolia & 1.8 & 75.7 & 0.1 & 23.3 & 0.9 & n.d. & n.d. \\
\hline raimondii & 7.6 & 74.5 & 10.2 & 14.4 & 0.9 & n.d. & 0.05 \\
\hline \multicolumn{8}{|l|}{ Thyrsiflorae } \\
\hline thyrsiflora & 5.1 & 2.9 & 94.3 & 0.8 & 2.0 & n.d. & 0.04 \\
\hline \multicolumn{8}{|l|}{ Rusticae } \\
\hline rustica & 5.4 & 98.2 & 0.5 & 0.8 & 0.5 & n.d. & n.d. \\
\hline \multicolumn{8}{|l|}{ Tabacum } \\
\hline \multicolumn{8}{|l|}{ Tomentosae } \\
\hline tomentosa & 1.1 & 4.4 & 79.0 & 2.8 & 13.8 & n.d. & 0.01 \\
\hline tomentosiformis & 0.5 & 6.0 & 65.0 & 4.7 & 24.3 & n.d. & 0.01 \\
\hline otophora & 0.4 & 7.3 & 74.8 & 4.1 & 13.8 & n.d. & n.d. \\
\hline setchellii & 4.2 & 2.6 & 92.4 & 2.8 & 2.2 & n.d. & 0.33 \\
\hline glutinosa & 7.4 & 18.9 & 77.4 & 1.4 & 2.3 & n.d. & 0.31 \\
\hline kawakamii & 0.3 & 14.0 & 73.2 & 2.2 & 10.6 & n.d. & n.d. \\
\hline \multicolumn{8}{|l|}{ Genuinae } \\
\hline tabacum (cv. NC 2326) & 6.1 & 95.1 & 1.1 & 0.4 & 3.4 & n.d. & n.d. \\
\hline \multicolumn{8}{|l|}{ Petunioides } \\
\hline \multicolumn{8}{|l|}{ Undulatae } \\
\hline undulata & 6.8 & 97.4 & 2.4 & 0.2 & n.d. & n.d. & n.d. \\
\hline arentsii & 7.5 & 93.2 & 1.5 & 4.3 & 1.0 & n.d. & n.d. \\
\hline wigandioides & 4.7 & 92.3 & 0.5 & 6.0 & 1.2 & n.d. & n.d. \\
\hline \multicolumn{8}{|l|}{ Trigonophyllae } \\
\hline trigonophylla & 1.2 & 2.8 & 92.4 & 1.9 & 2.9 & n.d. & 0.10 \\
\hline palmeri ${ }^{\star *}$ & 2.1 & 3.9 & 92.2 & 2.7 & 1.2 & n.d. & 0.24 \\
\hline \multicolumn{8}{|l|}{ Alatae } \\
\hline sylvestris & 4.8 & 82.2 & 16.9 & 0.4 & 0.5 & n.d. & 0.08 \\
\hline langsdorffii & 3.1 & 98.4 & 0.7 & 0.4 & 0.5 & n.d. & n.d. \\
\hline alata & 0.2 & 68.8 & 9.5 & 21.3 & n.d. & 0.4 & n.d. \\
\hline forgetiana & 0.1 & 90.8 & 6.7 & 2.5 & n.d. & n.d. & n.d. \\
\hline bonariensis & 0.3 & 88.1 & 7.8 & 4.1 & n.d. & n.d. & n.d. \\
\hline longiflora & 0.6 & 8.7 & 90.6 & n.d. & 0.7 & n.d. & n.d. \\
\hline plumbaginifolia & 0.9 & 17.3 & 77.6 & 3.6 & 1.5 & n.d. & 0.07 \\
\hline sanderae $^{\star *}$ & 0.1 & 88.7 & 4.6 & 6.7 & n.d. & n.d. & n.d. \\
\hline \multicolumn{8}{|l|}{ Repandae } \\
\hline repanda & 1.8 & 6.8 & 73.1 & 14.3 & 5.8 & n.d. & 1.03 \\
\hline stocktonii & 12.4 & 91.3 & 6.5 & 0.4 & 1.8 & n.d. & 0.69 \\
\hline nesophila & 7.5 & 9.5 & 86.7 & 1.0 & 2.8 & n.d. & 2.75 \\
\hline \multicolumn{8}{|l|}{ Noctiflorae } \\
\hline noctiflora & 10.7 & 0.6 & 16.8 & 76.6 & 6.0 & n.d. & 0.02 \\
\hline petunioides & 5.4 & 0.9 & 13.0 & 83.3 & 2.8 & n.d. & trace \\
\hline acaulis & 1.1 & 10.0 & 39.5 & 41.5 & 9.0 & n.d. & n.d. \\
\hline
\end{tabular}


Table 1. (cont'd.)

Alkaloid levels and composition of the Nicotiana species, from greenhouse-grown plants.

\begin{tabular}{c|c|c|c|c|c|c|c}
\hline \multirow{2}{*}{$\begin{array}{c}\text { Subgenus } \\
\begin{array}{c}\text { Section } \\
\text { Species }\end{array}\end{array}$} & $\begin{array}{c}\text { Total } \\
\text { alkaloids* } \\
(\mathrm{mg} / \mathrm{g})\end{array}$ & $\begin{array}{c}\text { Nico- } \\
\text { tine }\end{array}$ & $\begin{array}{c}\text { Nornico- } \\
\text { tine }\end{array}$ & $\begin{array}{c}\text { Anab- } \\
\text { asine }\end{array}$ & $\begin{array}{c}\text { Anat- } \\
\text { abine }\end{array}$ & $\begin{array}{c}\text { Myos- } \\
\text { mine }\end{array}$ & $\begin{array}{c}\text { N-acetyl- } \\
\text { nornicotine } \\
(\mathrm{mg} / \mathrm{g})\end{array}$ \\
\hline
\end{tabular}

Petunioides (cont'd.)

\begin{tabular}{|c|c|c|c|c|c|c|c|}
\hline \multicolumn{8}{|l|}{ Acuminatae } \\
\hline acuminata & 0.2 & 47.1 & 19.1 & 33.8 & n.d. & n.d. & n.d. \\
\hline pauciflora & 0.4 & 84.6 & 9.2 & 6.2 & n.d. & n.d. & n.d. \\
\hline attenuata & 9.0 & 96.2 & 1.6 & 0.7 & 0.9 & 0.6 & 0.01 \\
\hline miersii & 1.4 & 44.2 & 54.4 & 1.4 & n.d. & n.d. & n.d. \\
\hline corymbosa & 4.8 & 98.7 & 0.7 & trace & 0.6 & trace & n.d. \\
\hline linearis & 0.3 & 89.5 & 10.5 & n.d. & n.d. & n.d. & n.d. \\
\hline spegazzinii & 0.1 & 91.0 & 9.0 & n.d. & n.d. & n.d. & 0.07 \\
\hline \multicolumn{8}{|l|}{ Bigelovianae } \\
\hline bigelovii & 12.4 & 98.0 & 0.5 & 0.1 & 1.4 & trace & n.d. \\
\hline clevelandii & 15.7 & 99.1 & 0.2 & 0.1 & 0.6 & trace & n.d. \\
\hline \multicolumn{8}{|l|}{ Nudicaules } \\
\hline nudicaulis & 1.0 & 2.4 & 51.0 & 38.8 & 7.8 & n.d. & 0.04 \\
\hline \multicolumn{8}{|l|}{ Suaveolentes } \\
\hline suaveolens & 5.2 & 60.1 & 31.8 & 6.6 & 1.5 & n.d. & 0.07 \\
\hline exigua & 5.7 & 55.8 & 35.5 & 5.6 & 3.1 & n.d. & 0.06 \\
\hline maritima & 1.1 & 3.8 & 67.6 & 25.2 & 3.4 & n.d. & 0.02 \\
\hline velutina & 6.2 & 1.0 & 83.8 & 13.2 & 2.0 & n.d. & 0.13 \\
\hline amplexicaulis & 16.6 & 99.2 & 0.2 & 0.1 & 0.5 & n.d. & n.d. \\
\hline gossei & 6.4 & 97.4 & 0.7 & 1.2 & 0.7 & n.d. & n.d. \\
\hline benthamiana & 15.8 & 90.4 & 0.2 & 8.4 & 1.0 & n.d. & 1.16 \\
\hline excelsior & 19.1 & 96.7 & 0.2 & 1.9 & 1.2 & n.d. & trace \\
\hline goodspeedii & 3.1 & 3.7 & 49.5 & 45.2 & 1.6 & n.d. & 0.10 \\
\hline ingulba & 10.2 & 94.2 & 4.7 & 1.0 & 0.1 & n.d. & trace \\
\hline megalosiphon & 7.0 & 1.7 & 71.9 & 25.6 & 0.8 & n.d. & 0.02 \\
\hline rosulata & 1.2 & 33.7 & 60.6 & 5.2 & 0.5 & n.d. & 0.04 \\
\hline simulans & 0.4 & 25.6 & 63.8 & 9.2 & 1.4 & n.d. & n.d. \\
\hline hesperis & 3.4 & 80.8 & 10.4 & 7.3 & 1.5 & n.d. & n.d. \\
\hline occidentalis & 0.2 & 25.8 & 43.8 & 29.4 & 1.0 & n.d. & trace \\
\hline rotundifolia & 1.5 & 93.7 & 0.3 & 5.2 & 0.8 & n.d. & n.d. \\
\hline africana & 4.5 & 27.4 & 65.2 & 0.8 & 6.6 & n.d. & 0.07 \\
\hline cavicola & 1.3 & 10.4 & 69.6 & 17.7 & 2.3 & n.d. & n.d. \\
\hline umbratica & 0.2 & 36.8 & 48.6 & 13.6 & 1.0 & n.d. & n.d. \\
\hline debneyi & 5.6 & 40.8 & 5.2 & 48.6 & 5.4 & n.d. & n.d. \\
\hline fragrans & 3.2 & 97.6 & 0.1 & 0.1 & 2.2 & n.d. & n.d. \\
\hline
\end{tabular}

All of the species produced nicotine and nornicotine when grown either in the greenhouse or field. All species except $N$. spegazzinii contained anabasine when grown in the greenhouse, although under field conditions several species failed to produce detectable levels of anabasine. Measurable amounts of anatabine were also found in most species; however, several species failed to produce this alkaloid under either field or greenhouse environments. Myosmine was detected in only a few species.

A single alkaloid type made up the greatest portion of the total-alkaloid fraction in a majority of the species. The distribution between predominantly nicotine producers and predominantly nornicotine producers is about equal. Four species produce mainly anabasine, $N$. glauca, N. noctiflora, $N$. petunioides and $N$. debneyi. Anatabine and myosmine were never found to be the predominant alkaloids.

While one alkaloid was found to predominate for a given species, often a second alkaloid accounted for a significant portion of the remainder. The most frequent combinations of primary and secondary alkaloids was 
Table 2.

Alkaloid levels and composition of the Nicotiana species, from field-grown plants.

\begin{tabular}{|c|c|c|c|c|c|c|}
\hline \multirow{2}{*}{$\begin{array}{l}\text { Subgenus } \\
\text { Section } \\
\text { Species }\end{array}$} & \multirow{2}{*}{$\begin{array}{c}\text { Total } \\
\text { alkaloids* } \\
(\mathrm{mg} / \mathrm{g})\end{array}$} & \multicolumn{4}{|c|}{ Percentage composition } & \multirow{2}{*}{$\begin{array}{c}N \text {-acetyl- } \\
\text { nornicotine } \\
(\mathrm{mg} / \mathrm{g})\end{array}$} \\
\hline & & $\begin{array}{l}\text { Nico- } \\
\text { tine }\end{array}$ & $\begin{array}{l}\text { Nornico- } \\
\text { tine }\end{array}$ & $\begin{array}{l}\text { Anab- } \\
\text { asine }\end{array}$ & $\begin{array}{l}\text { Anat- } \\
\text { abine }\end{array}$ & \\
\hline
\end{tabular}

Rustica

Paniculatae

paniculata

knightiana

solanifolia

benavidesii

cordifolia

raimondii

\subsection{6}

12.1

1.2

86.7

n.d. ${ }^{+}$

0.04

$3.32 \quad 90.3$

92.7

8.3

0.3

1.1

0.7

n.d

$6.72 \quad 1.3$

57.3

1.7

81.9

$\begin{array}{ll}8.03 & 74.2\end{array}$

1.7

41.2

15.5

20.9

27.36

80.6

0.2

0.2

0.9

4.7

1.0

0.10

n.d.

n.d.

0.03

Thyrsiflorae

thyrsiflora

13.98

5.2

92.7

0.8

1.3

Rusticae

rustica

25.60

97.1

0.7

0.5

1.7

trace ${ }^{++}$

\section{Tabacum}

Tomentosae
tomentosa
tomentosiformis
otophora
setchellii
glutinosa
kawakamii

Genuinae

tabacum (cv. NC 2326)

\section{Petunioides}

Undulatae
undulata
arentsii
wigandioides
Trigonophyllae
trigonophylla
palmeri**
Alatae
sylvestris
langsdorffii
alata
forgetiana
bonariensis
longiflora
plumbaginifolia
sanderae ${ }^{\star *}$
Repandae
repanda
stocktonii
nesophila
Noctiflorae
noctiflora
petunioides
acaulis

6.80
0.63
0.95
6.29
7.35
1.14

\section{9}

7.1

84.2

69.9

81.8

8.1

2.7

87.8

85.4

11.3

78.5

13.00

95.0

1.6

0.4

3.0

n.d.

0.10

0.01

0.02

0.07

n.d.

0.02

12.87

28.70

8.30

1.20

4.68

14.23

1.65

0.09

0.07

0.28

0.19

1.82

5.58

14.32

18.13

18.9

88.3

14.2
7.0

2.9

0.4

83.3

89.8

3.6

\section{7}

n.d.

n.d.

50.7

40.1

87.9

-

64.5

8.6

81.9

17.00

3.15

1.08
1.0

1.4

6.9
18.6

10.9
1.0

6.4

4.8

0.4

0.6

1.5

0.8

1.1

4.0

3.1

n.d.

0.05

$0.3 \quad 1.6$

0.01

n.d.

n.d.

12.5

13.0

n.d.

0.8

\section{1}

n.d.

n.d.

n.d.

5.4

1.5

-

\section{2}

8.4

1.28

0.3

2.8

0.90

$\begin{array}{lll}1.4 & 2.5 & 2.24\end{array}$

71.6

8.8

n.d.

83.1

24.4

4.6
18.8

n.d.

0.02 
Table 2. (cont'd.)

Alkaloid levels and composition of the Nicotiana species, from field-grown plants.

\begin{tabular}{|c|c|c|c|c|c|c|}
\hline \multirow{2}{*}{$\begin{array}{l}\text { Subgenus } \\
\text { Section } \\
\text { Species }\end{array}$} & \multirow{2}{*}{$\begin{array}{c}\text { Total } \\
\text { alkaloids* } \\
(\mathrm{mg} / \mathrm{g})\end{array}$} & \multicolumn{4}{|c|}{ Percentage composition } & \multirow{2}{*}{$\begin{array}{c}\mathrm{N} \text {-acetyl- } \\
\text { nornicotine } \\
(\mathrm{mg} / \mathrm{g})\end{array}$} \\
\hline & & $\begin{array}{l}\text { Nico- } \\
\text { tine }\end{array}$ & $\begin{array}{l}\text { Nornico- } \\
\text { tine }\end{array}$ & $\begin{array}{l}\text { Anab- } \\
\text { asine }\end{array}$ & $\begin{array}{l}\text { Anat- } \\
\text { abine }\end{array}$ & \\
\hline
\end{tabular}

Petunioides (cont'd.)

\begin{tabular}{|c|c|c|c|c|c|c|}
\hline \multicolumn{7}{|l|}{ Acuminatae } \\
\hline acuminata & 0.14 & 61.9 & 38.1 & n.d. & n.d. & 0.02 \\
\hline pauciflora & 0.13 & 100.0 & n.d. & n.d. & n.d. & n.d. \\
\hline attenuata & 3.67 & 95.8 & 0.3 & 0.8 & 3.1 & 0.02 \\
\hline miersii & 2.00 & 89.3 & 10.7 & n.d. & n.d. & n.d. \\
\hline corymbosa & 2.01 & 98.5 & 0.9 & n.d. & 0.6 & 0.01 \\
\hline linearis & 0.13 & 92.3 & 7.7 & n.d. & n.d. & n.d. \\
\hline spegazzinii & 0.41 & 39.0 & 48.8 & 4.9 & 7.3 & 0.01 \\
\hline \multicolumn{7}{|l|}{ Bigelovianae } \\
\hline bigelovii & 7.76 & 94.1 & 1.0 & n.d. & 4.9 & n.d. \\
\hline clevelandii & 6.40 & 97.6 & 0.2 & n.d. & 2.2 & n.d. \\
\hline \multicolumn{7}{|l|}{ Nudicaules } \\
\hline nudicaulis & 2.47 & 7.4 & 65.5 & 18.3 & 8.8 & n.d. \\
\hline \multicolumn{7}{|l|}{ Suaveolentes } \\
\hline suaveolens & 3.09 & 59.8 & 32.8 & 5.2 & 2.2 & 0.01 \\
\hline exigua & 0.35 & 37.1 & 52.9 & 10.0 & n.d. & n.d. \\
\hline maritima & 1.73 & 5.6 & 73.7 & 14.6 & 6.1 & 0.06 \\
\hline velutina & 1.54 & 9.0 & 78.3 & 10.5 & 2.2 & 0.05 \\
\hline amplexicaulis & 5.62 & 98.1 & 0.1 & 0.1 & 1.7 & n.d. \\
\hline gossei & 17.55 & 96.0 & 0.5 & 1.2 & 2.3 & n.d. \\
\hline benthamiana & 7.69 & 82.5 & 1.0 & 12.5 & 4.0 & n.d. \\
\hline excelsior & 12.80 & 94.9 & n.d. & 1.0 & 4.1 & n.d. \\
\hline goodspeedii & 4.33 & 5.0 & 65.3 & 24.6 & 5.1 & 0.04 \\
\hline ingulba & 13.28 & 96.4 & 1.6 & 1.3 & 0.7 & n.d. \\
\hline megalosiphon & 5.12 & 4.1 & 70.6 & 22.8 & 2.5 & 0.12 \\
\hline rosulata & 2.71 & 17.9 & 75.3 & 4.4 & 2.4 & 0.01 \\
\hline simulans & 0.30 & 19.2 & 70.8 & 6.9 & 3.1 & 0.01 \\
\hline hesperis & 3.55 & 78.3 & 14.2 & 5.0 & 2.5 & n.d. \\
\hline occidentalis & 0.35 & 17.4 & 58.0 & 20.0 & 4.6 & 0.01 \\
\hline rotundifolia & 4.44 & 90.6 & 0.2 & 6.4 & 2.8 & 0.01 \\
\hline africana & 16.06 & 24.8 & 69.2 & n.d. & 6.0 & 0.18 \\
\hline cavicola & 0.42 & 20.9 & 60.5 & 16.3 & 2.3 & n.d. \\
\hline umbratica & 0.11 & 39.3 & 50.9 & 9.8 & n.d. & n.d. \\
\hline debneyi & 2.22 & 34.9 & 12.5 & 45.9 & 6.7 & n.d. \\
\hline fragrans & 7.81 & 95.0 & 0.5 & 1.2 & 3.3 & 0.01 \\
\hline
\end{tabular}

* Average of 1985 and 1986 (two replications per year).

** Tentative species status.

+ n.d.: none detected.

++ Detected but below $0.01 \mathrm{mg} / \mathrm{g}$.

nicotine-nornicotine followed closely by nornicotinenicotine. Nicotine-anabasine was the only other combination which occurred in any frequency. Anabasine and anatabine did not occur together in a predominant combination in any of the species.

Among the species with the highest total-alkaloid levels, nicotine frequently accounted for nearly the entire alkaloid fraction. Only in $N$. noctiflora, where anabasine accounts for 75 percent of the total-alkaloid composition, did an alkaloid other than nicotine comprise the major fraction of a high alkaloid-producing species.

Results comparing the alkaloid composition of green, freeze-dried samples with air-dried samples are presented in Table 3. Eleven of the 25 species from which air-dried samples were produced were found to have a different alkaloid composition from that of the greenleaf sample. In most cases, curing resulted in an increased proportion of nornicotine at the apparent expense of nicotine which decreased, indicating these species have the so-called converter trait (6). 
Table 3.

Alkaloid composition in green and cured lamina of Nicotiana species with the ability to convert nicotine to nornicotine.

\begin{tabular}{|c|c|c|c|c|c|c|c|c|c|}
\hline \multirow{3}{*}{ Nicotiana species } & \multirow{3}{*}{ Sample type } & \multicolumn{8}{|c|}{ Percentage composition } \\
\hline & & \multicolumn{4}{|c|}{ Greenhouse (1984) } & \multicolumn{4}{|c|}{ Field (1986) } \\
\hline & & $\begin{array}{l}\text { Nico- } \\
\text { tine }\end{array}$ & $\begin{array}{c}\text { Nornico- } \\
\text { tine }\end{array}$ & $\begin{array}{l}\text { Anab- } \\
\text { asine }\end{array}$ & $\begin{array}{l}\text { Anat- } \\
\text { abine }\end{array}$ & $\begin{array}{l}\text { Nico- } \\
\text { tine }\end{array}$ & $\begin{array}{c}\text { Nornico- } \\
\text { tine }\end{array}$ & $\begin{array}{l}\text { Anab- } \\
\text { asine }\end{array}$ & $\begin{array}{l}\text { Anat- } \\
\text { abine }\end{array}$ \\
\hline \multirow[t]{2}{*}{ forgetiana } & green (freeze-dried) & 82.4 & 12.8 & 4.7 & n.d. ${ }^{*}$ & 100.0 & n.d. & n.d. & n.d. \\
\hline & air-cured & 58.6 & 37.5 & 2.9 & 1.0 & 50.0 & n.d. & 50.0 & n.d. \\
\hline \multirow[t]{2}{*}{ glutinosa } & green (freeze-dried) & 19.5 & 75.9 & 1.4 & 3.2 & 84.0 & 12.9 & 0.3 & 2.8 \\
\hline & air-cured & - & - & - & - & 1.0 & 88.1 & 7.0 & 3.9 \\
\hline \multirow[t]{2}{*}{ gossei } & green (freeze-dried) & 97.4 & 1.0 & 1.2 & 0.4 & 95.4 & 0.5 & 1.5 & 2.6 \\
\hline & air-cured & 27.2 & 69.4 & 2.9 & 0.6 & 67.8 & 24.8 & 3.7 & 3.7 \\
\hline \multirow[t]{2}{*}{ hesperis } & green (freeze-dried) & 82.0 & 10.0 & 6.6 & 1.4 & 77.4 & 14.9 & 5.1 & 2.7 \\
\hline & air-cured & 1.8 & 79.9 & 16.6 & 1.7 & 35.6 & 49.6 & 11.3 & 3.6 \\
\hline \multirow[t]{2}{*}{ ingulba } & green (freeze-dried) & 95.4 & 3.6 & 0.9 & $<0.1$ & 96.8 & 1.5 & 1.0 & 0.7 \\
\hline & air-cured & 12.8 & 80.2 & 7.0 & n.d. & 26.5 & 67.2 & 6.2 & 0.1 \\
\hline \multirow[t]{2}{*}{ knightiana } & green (freeze-dried) & 93.7 & 3.7 & 2.5 & 0.2 & 90.7 & 6.3 & 2.3 & 0.7 \\
\hline & air-cured & 49.1 & 31.3 & 18.7 & 0.9 & 53.4 & 26.0 & 15.1 & 5.5 \\
\hline \multirow[t]{2}{*}{ paniculata } & green (freeze-dried) & 84.9 & 11.3 & $\operatorname{trace}^{\star \star}$ & 0.3 & 90.1 & 8.3 & 1.1 & 2.8 \\
\hline & air-cured & - & - & - & - & 3.6 & 89.9 & 1.8 & 4.7 \\
\hline \multirow[t]{2}{*}{ stocktonii } & green (freeze-dried) & 90.1 & 7.6 & 0.3 & 1.9 & 84.2 & 6.6 & 0.3 & 1.2 \\
\hline & air-cured & 80.0 & 15.8 & 1.1 & 3.2 & 39.6 & 53.8 & 3.8 & 2.8 \\
\hline \multirow[t]{2}{*}{ suaveolens } & green (freeze-dried) & 55.9 & 35.0 & 7.9 & 2.2 & 65.9 & 26.9 & 4.6 & 2.6 \\
\hline & air-cured & - & - & - & - & 9.8 & 69.4 & 17.6 & 3.2 \\
\hline \multirow[t]{2}{*}{ sylvestris } & green (freeze-dried) & 68.4 & 30.7 & 0.5 & 0.4 & 92.2 & 5.2 & 0.5 & 2.2 \\
\hline & air-cured & 31.1 & 61.3 & 6.2 & 1.3 & 2.7 & 91.9 & 2.8 & 2.2 \\
\hline \multirow[t]{2}{*}{ undulata } & green (freeze-dried) & 96.7 & 3.3 & n.d. & n.d. & 93.3 & 5.3 & 1.0 & 0.3 \\
\hline & air-cured & 74.9 & 23.0 & 2.1 & n.d. & 78.9 & 13.6 & 5.7 & 1.8 \\
\hline
\end{tabular}

* n.d.: none detected.

** Detected but below $0.01 \mathrm{mg} / \mathrm{g}$. 


\section{DISCUSSION}

This study represents the most recent effort to characterize the alkaloid content of the species in the Nicotiana genus. The results are unique in that the alkaloid content of all available Nicotiana species is reported. Moreover, these analyses were conducted on multiple samples produced over a four-year period and provide a cumulative comparison of greenhouse and field environments. These results provide one of the most thorough quantitative measures of individual nicotine alkaloids occurring in each species to date.

As in other reports $(4,9,10,11,15,16)$, alkaloid levels and composition were highly variable among the Nicotiana species. For the genus as a whole, there were no immediately obvious associations between alkaloid characteristics and geographic origin, morphology, or chromosome number. There do, however, appear to be similarities in alkaloid composition, and, to a lesser extent, in total-alkaloid levels within the various taxonomic sections of the genus. For example, in the section Paniculatae, total-alkaloid levels are in the medium range relative to the other 13 taxonomic sections. Five of the seven species in this section produce mainly nicotine. Of the two exceptions, $N$. glauca produces anabasine in the greatest quantity, while $N$. solanifolia produces equal amounts of anabasine and nornicotine. GOODSPEED (2) indicates that the morphological and cytological distinctions of $N$. glauca within this section point to a separate evolution, which could easily account for the differences in alkaloid make-up. This is not the case for $N$. solanifolia. This species shows a close morphological and cytological association with other members of the section and an explanation of its rather unique alkaloid profile is not readily apparent. Low and medium total-alkaloid levels characterize the species in section Tomentosae. A member of this section is believed to be one of the progenitors of cultivated tobacco, N. tabacum. Nornicotine is the predominant alkaloid in all six species, which includes $N$. kawakamii, the most recently described species in the genus (7). Analyses of $N$. glutinosa from the greenhouse and field were somewhat contradictory. While this species produced the highest level of alkaloids in this section in both environments, the predominant alkaloid was nornicotine in the greenhouse and nicotine in the field (Tables 1 and 2). An analysis of a cured-leaf sample from the greenhouse was not made; however, a curedleaf sample from the field was found to contain mainly nornicotine (Table 3). N. glutinosa has a number of morphological characteristics in common with members of the section Paniculatae (2). It is feasible that this association is also reflected in its plant chemistry and accounts for some of the differences in alkaloid production by this species. The need for further indepth evaluation is suggested.

In the section Alatae, total-alkaloid levels are low, except for $N$. sylvestris and possibly $N$. langsdorffii, which have levels more in the medium to high range. Six of the eight species in this section produce predom- inantly nicotine. The main alkaloid in $N$. longiflora and $N$. plumbaginifolia is nornicotine, although it should be noted that total-alkaloid levels are very low in these species. The species in this section can be grouped into 9, 10, and 12-paired chromosome members. N. longiflora and $N$. plumbaginifolia are the only two species in this section with 10-chromosome pairs. This suggests that while the chromosome number may not be directly associated with the alkaloid content over the entire genus, relationships within phylogenetic groups probably exist.

Another example of phylogenetic associations was observed in the section Noctiflorae. This section consists of four species; however, one of these species, $N$. ameghinoi, is known only from previous herbarium specimens and was not available for testing. The three species which were tested are characterized by the predominance of anabasine despite the fact that their totalalkaloid levels are highly variable.

The section Suaveolentes is the largest section in the genus with 21 species. With the exception of $N$. fragrans, which is found on several small South Pacific islands, and $N$. africana, recently discovered in South Africa, the remaining 19 species are endemic to the Australian continent. This section exhibits a wide range in alkaloid levels, which encompass the extremes of the genus. Similarly, alkaloid composition is quite heterogeneous among the members of this section. Despite the variation exhibited in the Suaveolentes section, certain tendencies in alkaloid levels and distribution are apparent. The section can be subdivided into three basic groups (Figure 1). The first group includes the species $N$. gossei $(2 \mathrm{n}=36), N$. amplexicaulis $(2 \mathrm{n}=36), N$. benthamiana $(2 \mathrm{n}=38), N$. excelsior $(2 \mathrm{n}=38)$, and $N$. ingulba $(2 n=40)$. This group is found in the arid central and northern regions of the continent. Total-alkaloid levels are high for all five species and among the highest in the genus. Nicotine accounts for over 90 percent of the total alkaloids in all of these species.

A second grouping of the Australian species contains $N$. suaveolens $(2 \mathrm{n}=32), N$. maritima $(2 \mathrm{n}=32), N$. velutina $(2 \mathrm{n}=32), N$. exigua $(2 \mathrm{n}=32), N$. megalosiphon $(2 \mathrm{n}=40), N$. goodspeedii $(2 \mathrm{n}=40)$, and $N$. debneyi $(2 n=48)$. These species grow primarily in the southcentral and eastern regions of the continent. Total-alkaloid levels are in the medium range. Nornicotine is the predominant alkaloid in this group; however, nicotine and anabasine occur in significant or major proportions in some of the species.

The third group includes $N$. simulans $(2 \mathrm{n}=40), N$. rosulata $(2 n=40), N$. occidentalis $(2 n=42), N$. hesperis $(2 n=42), \quad N$. rotundifolia $(2 n=44), N$. umbratica $(2 \mathrm{n}=46)$, and $N$. cavicola $(2 \mathrm{n}=46)$. The seven species are found primarily in western Australia, typically near the coast. Alkaloid levels for this group are in the low range. The alkaloid distribution in these species is somewhat variable, but nicotine and nornicotine make up the greatest proportion of alkaloids found.

When grouped in this manner it appears that the different kinds and relative amounts of alkaloids of the spe- 
Table 4.

Nicotiana species for which differences in the identification of the major alkaloid have been reported in independent investigations.

\begin{tabular}{|c|c|c|c|c|c|c|c|}
\hline \multirow[b]{2}{*}{$\begin{array}{l}\text { Nicotiana } \\
\text { species }\end{array}$} & \multicolumn{7}{|c|}{ Reference } \\
\hline & $\begin{array}{c}\text { SISSON \& } \\
\text { SEVERSON } \\
(1989)\end{array}$ & $\begin{array}{l}\text { No. } 10 \\
\text { SARYCHEV } \\
\text { et al. } \\
(1985)\end{array}$ & $\begin{array}{l}\text { No. } 9 \\
\text { SAITOH } \\
\text { et al. } \\
(1985)\end{array}$ & $\begin{array}{c}\text { No. } 15 \\
\text { SMITH \& } \\
\text { ABASHIAN } \\
(1963)\end{array}$ & $\begin{array}{c}\text { No. } 4 \\
\text { JEFFREY } \\
\text { (1959) }\end{array}$ & $\begin{array}{l}\text { No. } 16 \\
\text { SMITH \& } \\
\text { SMITH } \\
(1942)\end{array}$ & $\begin{array}{c}\text { No. } 11 \\
\text { SHMUCK \& } \\
\text { BOROZDINA } \\
(1941)\end{array}$ \\
\hline paniculata & nicotine & nicotine & nicotine & $\begin{array}{l}\text { nicotine, } \\
\text { nornicotine }\end{array}$ & $\begin{array}{l}\text { nicotine, } \\
\text { nornicotine }\end{array}$ & nornicotine & nicotine \\
\hline solanifolia & $\begin{array}{c}\text { nornicotine, } \\
\text { anabasine }\end{array}$ & $\begin{array}{l}\text { anabasine, } \\
\text { nornicotine }\end{array}$ & nornicotine & $\begin{array}{l}\text { nornicotine } \\
\text { anabasine }\end{array}$ & & & $\begin{array}{l}\text { nicotine, } \\
\text { nornicotine }\end{array}$ \\
\hline cordifolia & nicotine & nicotine & nicotine & anabasine & & & \\
\hline raimondii & nicotine & & nicotine & nicotine & & nornicotine & nicotine \\
\hline undulata & nicotine & nornicotine & nicotine & nicotine & nicotine & nornicotine & nicotine \\
\hline sylvestris & nicotine & nornicotine & nicotine & nornicotine & nicotine & nornicotine & nornicotine \\
\hline bonariensis & nicotine & & nicotine & nornicotine & & & nicotine \\
\hline longiflora & nornicotine & & nicotine & nicotine & nornicotine & nicotine & nornicotine \\
\hline sanderae & nicotine & nicotine & & & & & nornicotine \\
\hline stocktonii & nicotine & anabasine & nicotine & nornicotine & & nornicotine & \\
\hline pauciflora & nicotine & nicotine & nicotine & nornicotine & & & nornicotine \\
\hline corymbosa & nicotine & & nicotine & nornicotine & & & \\
\hline spegazzinii & nicotine & & nornicotine & & & & \\
\hline clevelandii & nicotine & nicotine & nicotine & anabasine & nicotine & & nicotine \\
\hline nudicaulis & $\begin{array}{l}\text { nornicotine, } \\
\text { anabasine }\end{array}$ & nornicotine & nornicotine & anabasine & nornicotine & nornicotine & \\
\hline suaveolens & nicotine & nicotine & nicotine & $\begin{array}{l}\text { nicotine, } \\
\text { anabasine }\end{array}$ & & nornicotine & nornicotine \\
\hline benthamiana & nicotine & nicotine & nicotine & anabasine & nicotine & & nornicotine \\
\hline goodspeedii & $\begin{array}{l}\text { nornicotine, } \\
\text { anabasine }\end{array}$ & & nornicotine & $\begin{array}{l}\text { nornicotine, } \\
\text { anabasine }\end{array}$ & & nornicotine & \\
\hline rotundifolia & nicotine & & nicotine & nicotine & nicotine & & anabasine \\
\hline
\end{tabular}


Figure 1.

Geographic distribution, chromosome number $(2 n)$ and total-alkaloid level (mg per g dry weight) of the Australian Nicotiana species (section Suaveolentes).

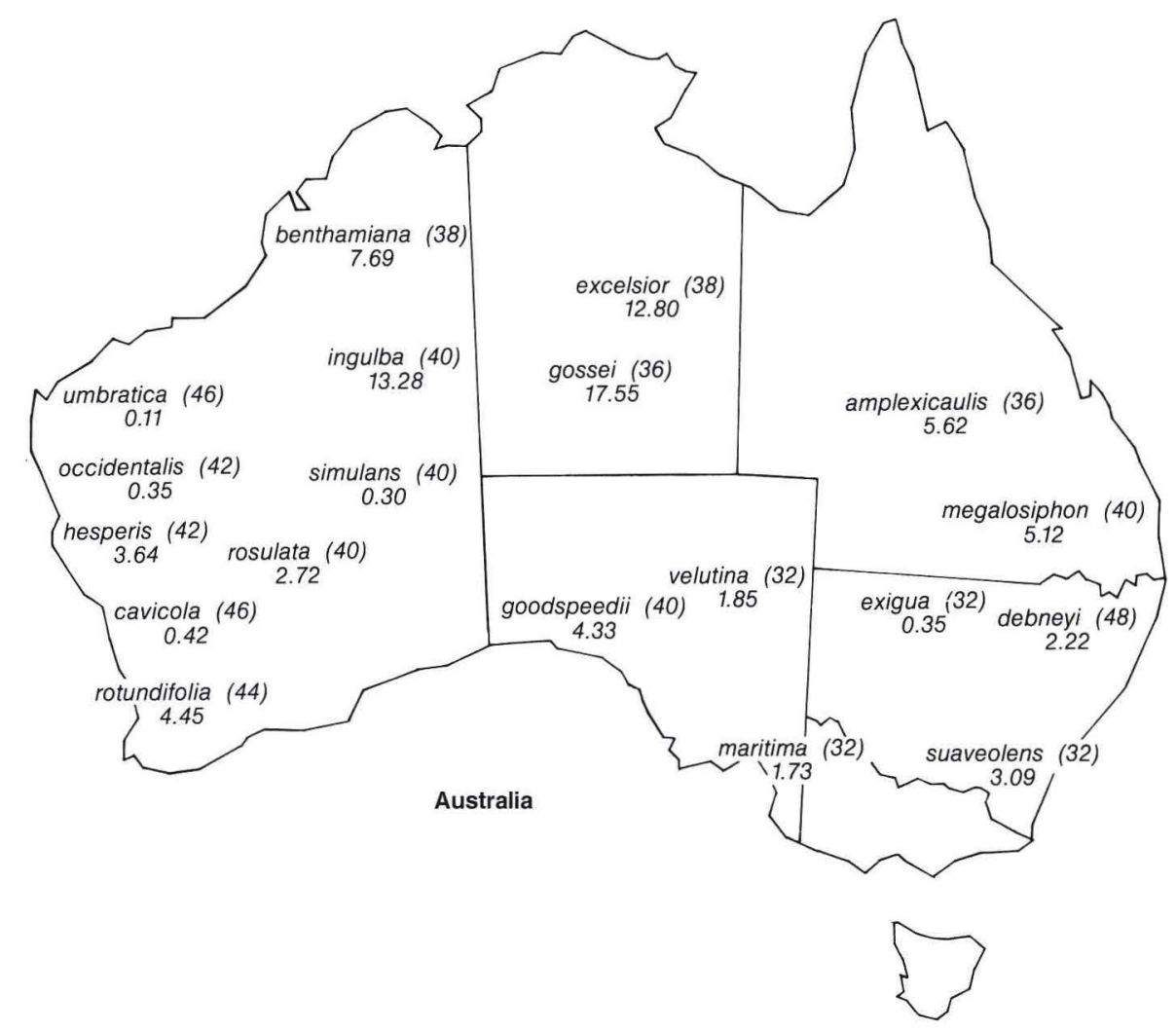

cies in the section Suaveolentes have an association with their geographic distribution. Geographic distribution is frequently confounded with evolutionary development. Goodspeed (2) hypothesized that the likely progenitors of this section, $N$. suaveolens and $N$. debneyi, became established in the southeastern portion of Australia and that hybridization and aneuploidy played major roles in the evolution and spread of this section westward. The section Suaveolentes contains a nearly complete aneuploid series from 16 to 24-paired chromosome species. Goodspeed (2) suggested that the present distribution of chromosome numbers in Australia reflects the survival value of certain genomic combinations. The high alkaloid-producing species which contain primarily nicotine are found only in the arid climate of the interior of the continent. Whether or not alkaloid content is related to the adaptation of these species to a specific environment poses an interesting question which is beyond the scope of this study.

The comparison of alkaloid composition between green, freeze-dried and air-cured samples (Table 3) proved informative. Our main interest was in detecting conversion of nicotine to nornicotine as previously reported (1). Eight species which produced mainly nicotine in the green-leaf sample were found to contain mostly nornicotine after air curing. These eight species were scattered in four of the 14 sections but four species belong to the Suaveolentes section. JEFFrey (4), who made a similar type comparison, also reported eight species which converted nicotine to nornicotine upon curing; however, only three of these, N. gossei, $N$. sylvestris, and $N$. undulata, were in common with our findings. No explanation for these inconsistencies is readily available, although growing and curing conditions and analytical procedures were different.

The synthesis and accumulation of nicotine, time and site of conversion, and the inheritance of conversion of nicotine to nornicotine in tobacco and related amphiploids has been reported $(1,3,5,6,18)$ and will not be reviewed. It is clear, however, that there are two systems or routes for conversion of nicotine to nornicotine among the Nicotiana species. Both systems are under genetic control. Nicotine, which is produced in the roots, is translocated to the leaf where it is rapidly converted to nornicotine by demethylation $(1,18)$. Alternatively, nicotine can be translocated to the leaf where it accumulates and conversion does not take place until the late stages of senescence or curing (6). Most of the species which are found to contain mainly nornicotine follow the first system of conversion.

As indicated, the alkaloid content of the Nicotiana species has been the subject of several previous investigations $(4,9,10,11,15,16)$. Duplicate analyses now exist for most species. Because of the diversity of growing conditions, sampling, and analytic methods, the comparison of absolute values would not be practical. 
However, general agreement is found in the identification of the predominant alkaloid in 43 of the 62 species for which more than one analysis is available. This consistency suggests that comparisons of alkaloid composition among previous reports should generally be compatible.

The discrepancies in the identification of the major alkaloids reported by various independent investigators is summarized in Table 4. The most frequent disparity in reporting was between nicotine and nornicotine. More than half (11 out of 19) of the differences involved these two alkaloids. As discussed by previous investigators, these differences may be due to the action of genetic factors for converting nicotine to nornicotine under certain cultural and curing conditions $(4,14)$. Other differences involved the identification between nicotine and anabasine and nornicotine versus anabasine. There is no evidence to suggest that these discrepancies are genetic in nature, although this element cannot be ruled out. There are, however, a number of other factors which may have influenced the results. Different analytical techniques were employed in each investigation. Cultural conditions varied from one experiment to another. Methods of sampling differed. Seed sources were different. The single or combined effects that each of these variables can have on the kind and amount of each alkaloid produced is not evident. However, comparison of these results does suggest that closely-controlled testing is needed in evaluating the alkaloid content of certain Nicotiana species.

The current study has not dramatically changed the conclusions of previous investigators concerning the alkaloid content of the Nicotiana species. Either nicotine or nornicotine is produced most frequently as the principal alkaloid, anabasine only rarely. In contrast to the report by SMITH and ABASHIAn (15), who found nicotine and nornicotine to be completely absent in some species, our results showed these two alkaloids present in all species. This likely reflects the greater sensitivity of our analytical methods. In the same study, they reported anabasine occurred as the principal alkaloid in 14 species. Although we found anabasine to be present in most species, it is doubtful it occurs as the major alkaloid with such regularity. Sмiтh and Aвashian's (15) results were based on visual estimates from paper chromatograms. These estimates are much more difficult to interpret quantitatively than those from GC analysis. We found anabasine as the principal alkaloid in only four species, $N$. glauca, $N$. noctiflora, $N$. petunioides, and $N$. debneyi, but anabasine is produced by most species.

These results substantiate many of the findings of earlier reports dealing with the alkaloid content of the $\mathrm{Ni}$ cotiana species. Environmental and cultural influences still pose the greatest sources of error in comparing our results with other studies. The precision of the analytical technique used in identifying individual alkaloid components and replicated sampling over years and environments makes this investigation unique. These results do provide more detailed and accurate information of the Nicotiana genus on an individual-species basis. These data establish a precedent for further studies of the genetics and evolution of alkaloid production among the Nicotiana species. They should also be of practical value to researchers in utilizing the wild species for improving tobacco germplasm.

\section{REFERENCES}

1. Dawson, R. F.: An experimental analysis of alkaloid production in Nicotiana - The origin of nornicotine; Am. J. Bot. 32 (1945) 416-423.

2. Goodspeed, T. H.: The genus Nicotiana; Chron. Bot., Vol. 16, Nos. 1-6 (1954) 1-536, Chronica Botanica Co., Waltham, Mass., U.S.A.

3. Griffith, R. B., W. D. Valleau and G. W. Stokes: Determination and inheritance of nicotine to nornicotine conversion in tobacco; Science (Wash., D.C.) 121 (1955) 343-344.

4. Jeffrey, R. N.: Alkaloid composition of species of Nicotiana; Tob. Sci. 3 (1959) 89-93.

5. Mann, T. J., and J. A. Weybrew: Inheritance of alkaloids in hybrids between flue-cured tobacco and related amphidiploids; Tob. Sci. 2 (1958) 29-34.

6. Mann, T. J., J. A. Weybrew, D. F. Matzinger and J. L. Hall: Inheritance of the conversion of nicotine to nornicotine in varieties of Nicotiana tabacum L. and related amphiploids; Crop Sci. 4 (1964) 349353.

7. Ogura, H.: Cytogenetic studies on a recently identified Nicotiana species, N. kawakamii OHAsHI; Cytologia (Tokyo) 45 (1980) 33-42.

8. Robinson, T.: Metabolism and function of alkaloids in plants; Science (Wash., D.C.) 184 (1974) 430435.

9. Saitoh, F., M. Noma and N. Kawashima: Alkaloid contents of sixty Nicotiana species; Phytochemistry (Oxf.) 24 (1985) 477-480.

10. Sarychev, Y. F., and N. A. Sherstyanykh: The pyridine bases of the genus Nicotiana; Tabak (Mosc.) No. 2, 1985, 6-12.

11. Shmuck, A. A., and A. Borozdina: Alkaloids of various plant species within the genus Nicotiana; C.R. (Doklady) Acad. Sci. URSS 32, No. 1 (1941) 62-65.

12. Severson, R. F., R. F. Arrendale, H. G. Cutler, D. Jones, V. A. Sisson and M. G. Stephenson: The chemistry and biological activity of acyl nornicotines from Nicotiana repandae; in Biologically active natural products - Potential use in agriculture, H. G. Cutler, ed., ACS (Am. Chem. Soc.) Symp. Ser. No. 380, pp. 335-362, American Chemical Society, Washington, D.C., 1988.

13. Severson, R. F., K. L. McDuffie, R. F. Arrendale, G. R. Gwynn, J. F. Chaplin and A. W. Johnson: Rapid method for the analysis of tobacco nicotine alkaloids; J. Chromatogr. 211 (1981) 111-121. 
14. Smith, H. H.: The genus as a genetic resource; in Nicotiana - Procedures for experimental use, R. D. Durbin, ed., U.S. Dep. Agric. Tech. Bull. 1586, pp. 1-16, 1979.

15. Smith, H. H., and D. V. Abashian: Chromatographic investigations on the alkaloid content of Nicotiana species and interspecific combinations; Am. J. Bot. 50 (1963) 435-447.

16. Smith, H. H., and C. R. Smith: Alkaloids in certain species and interspecific hybrids of Nicotiana; J. Agric. Res. 65 (1942) 347-359.

17. Tso, T. C.: Some novel concepts on the biosynthesis and biogenesis of tobacco alkaloids; Bot. Bull. Acad. Sin. (Taipei) 3 (1962) 61-71.

18. Wernsman, E. A., and D. F. Matzinger: Time and site of nicotine conversion in tobacco; Tob. Sci. 12 (1968) 226-228.
Addresses of authors:

Verne A. Sisson,

Crops Research Laboratory,

Agricultural Research Service, South Atlantic Area, U.S. Department of Agriculture,

Hillsboro Street Ext., P.O. Box 1555,

Oxford, North Carolina, 27565, U.S.A.

R. F. Severson,

Tobacco Quality and Safety Research Unit, Richard B. Russell Agricultural Research Center, Agricultural Research Service, U.S. Department of Agriculture,

College Station Road, P.O. Box 5677, Athens, Georgia, 30613, U.S.A. 